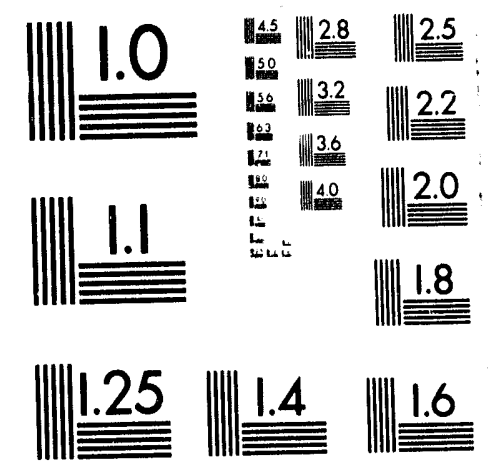



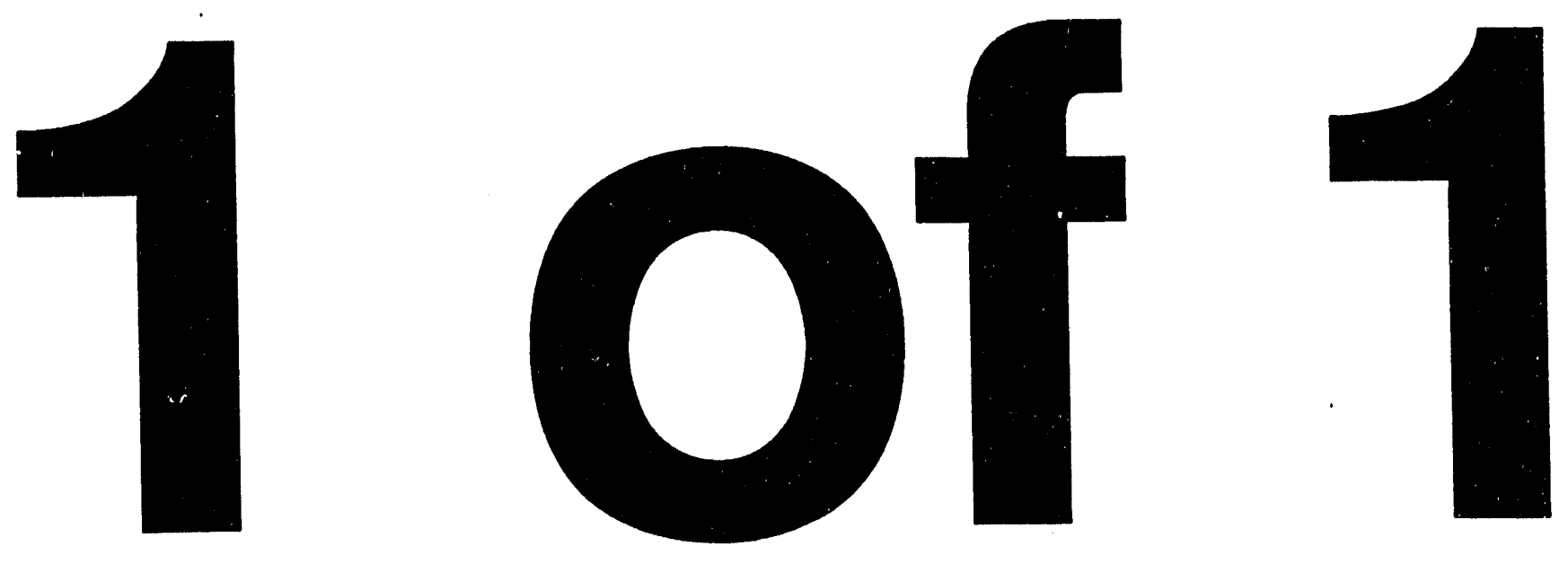


\title{
NON-MARKOVIAN QUANTUM BROWNIAN MOTION
}

\author{
OF A HARMONIC OSCILLATOR
}

\author{
J. TANG \\ Chemistry Division, Argonne National Laboratory \\ Argonne, IL 60439
}

\section{DISCLAIMER}

This report was prepared as an account of work sponsored by an agency of the United States Government. Neither the United States Government nor any agenc $j$ thereof, nor any of their employees, makes any warranty, express or implied, or assumes any legal liability or responsibility for the accuracy, completeness, or usefulness of any information, apparatus, product, or process disclosed, or represents that its use would not infringe privately owned rights. Reference herein to any specific commercial product, process, or service by trade name, trademark, manufacturer, or otherwise does not necessarily constitute or imply its endorsement, recommendation, or favoring by the United States Government or any agency thereof. The views and opinions of authors expressed herein do not necessarily state or reflect those of the United States Government or any agency thereof.

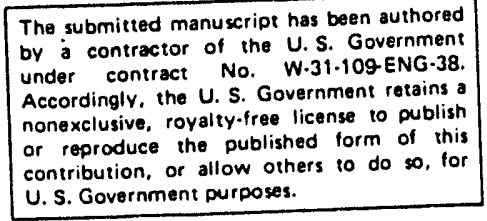




\section{Abstract}

We apply the density-matrix method to the study of quantum Brownian motion of a harmonic oscillator coupled to a heat bath, a system investigated previously by Caldeira and Leggett using a different method. Unlike the earlier work, in our derivation of the master equation the non-Markovian terms are maintained. Although the same model of interaction is used, discrepancy is found between their results and our equation in the Markovian limit. We also point out that the particular interaction model used by both works cannot lead to the phenomenological generalized Langevin theory of Kubo. 


\section{Introduction}

Any system in nature cannot sustain perpetual periodical motion indefinitely. Because of its constant interaction with the surrounding environment, dissipation always occurs. In the classical treatment, the phenomenological Langevin equation is introduced with a dissipative term to represent the frictional resistance imposed by the surrounding medium. The dissipative term in the Langevin equation is irreversible in time because of its dependence on velocity. How can one provide a quantum mechanical description of the time-irreversible dissipative force? Over the past three decades, there are some attempts to answer the question. Among them, Dekker [1] proposed a quantization procedure for complex canonical coordinates and momenta. $\mathrm{He}$ introduced a non-Hermitian Hamiltonian operator for the description of noiseinduced friction and was able to derive the Fokker-Planck equation for the Wigner transform of the density matrix. Because of the absence of knowledge concerning the details of the reservoir, the damping factor in his theory could not be related to the microscopic parameters associated with the heat bath. To overcome the difficulty of Dekker, Senitzky [2] suggested that one should take explicitly into account the interaction of the system and the reservoir as in the 
treatment of the spin relaxation by Bloch and Wangsness [3]. By including interaction of a spin with the quantized heat bath, the Bloch equation was derived with an expression for the relaxation rate valid at low temperatures. The method was generalized later by Zwanzig [4], Mori [5], Nakajima [6], and some similar approaches have also been used by many others.

Haake [7] showed that if one assumed the special type of interaction such as $a b^{+}+a^{+} b$, involving pairing-up of the creation $\left(a^{+}\right)$or the annihilation operators (a) for the system with $b$ and $b^{+}$for the bath, he could obtain a master equation equivalent to Dekker's result [1]. More recently, Caldeira and Leggett [8] used a different approach, the path integral method of Feynman and Vernon [9], also examined the quantum Brownian motion. In their work, the interaction is assumed to be linear in $\Sigma f_{k} p_{k} X$, where $X$ is the oscillator's coordinate and $\mathrm{p}_{\mathrm{k}}$ is the $\mathrm{k}$-th particles' momentum in the reservoir. Because a different type of interaction is assumed, their results are not exactly the same as the previous works $[1,7]$. In addition, in their derivation of the master equation and subsequent discussion the Markovian approximation was employed. 
In this report, we re-examine the quantum system of a damped oscillator interacting with a quantized bath at an arbitrary temperature, assuming the same type of linear interaction. The master equation for the time evolution of the density matrix is derived using the approach of Bloch and Wangsness [3]. Our derivation and discussion will be focused on the non-Markovian aspects and their consequences. The difference between our results and the work of Caldeira and Leggett will be discussed. We also point out that the particular interaction model suggested by them cannot lead to Kubo's phenomenological generalized Langevin equation [10].

\section{Derivation of the non-Markovian master equation}

We consider here a system consists of a harmonic oscillator, coupled to a surrounding medium (bath) with $\mathrm{N}(\mathrm{N}>>1)$ independent harmonic oscillators. The Hamiltonian for the total system is given by

$$
\begin{aligned}
H & =H_{S}+H_{B}+H_{I} \\
& =\frac{P^{2}}{2 M}+\frac{1}{2} M \Omega^{2} X^{2}+\sum_{k=1}^{N}\left[\frac{p_{k}^{2}}{2 m_{k}}+\frac{1}{2} m_{k} \omega_{k}^{2} q_{k}^{2}\right]+h X F .
\end{aligned}
$$

where $\mathrm{H}_{0}=\mathrm{H}_{\mathrm{S}}+\mathrm{H}_{\mathrm{B}}$, and the last term $\mathrm{H}_{\mathrm{I}}$ describes the interaction between 
the system and the bath. In Caldeira and Leggett's work [8] the bath operator $\mathrm{F}$ is given by $\Sigma \mathrm{f}_{\mathrm{k}} \mathrm{p}_{\mathrm{k}}$, but we consider $\mathrm{F}$ here either $\Sigma \mathrm{f}_{\mathrm{k}} \mathrm{p}_{\mathrm{k}}$ or $\Sigma \mathrm{f}_{\mathrm{k}} \mathrm{x}_{\mathrm{k}}$.

One can show that the equation for time evolution of $\hat{\rho}(t)$, the density matrix for the total system in the interaction representation, is given by

$$
\frac{d \hat{\rho}(t)}{d t}=-\frac{i}{\hbar}\left[\hat{H}_{I}(t), \hat{\rho}(0)\right]-\frac{1}{\hbar^{2}} \int_{0}^{t} d \tau\left[\hat{H}_{I}(t),\left[\hat{H}_{I}(t-\tau), \hat{\rho}(t-\tau)\right]\right],
$$

where

$$
\begin{aligned}
& \hat{\rho}(t)=e^{i H_{0} \omega n} \rho(t) e^{-H_{0} / m} \\
& \hat{H}_{I}(t)=e^{i H_{0} t h} H_{I} e^{-i H_{0} t h} .
\end{aligned}
$$

Eq. (2) is a formally exact integro-differential equation. Here we shall make the Born approximation [7] assuming the bath remains at thermal equilibrium all the time. We shall also assume that the bath is initially in thermal equilibrium and there is no correlation between $\mathrm{H}_{\mathrm{I}}$ and $\hat{\rho}(0)$, thus the first term in eq. (2) can be omitted. By taking an ensemble average of the bath, one can obtain the following equation for the reduced density matrix $\sigma(t)$, defined as $\operatorname{Tr}_{B}(\rho(t))$, 


$$
\begin{aligned}
& \frac{d \sigma(t)}{d t}=-\frac{i}{\hbar}\left[H_{S}, \sigma(t)\right] \\
& -\frac{1}{\hbar^{2}} \int_{0}^{t} d \tau \operatorname{Tr}_{B}\left(\left[H_{I},\left[e^{-i H_{0} \tau / h_{1}} H_{I} e^{i H_{0} \tau / \lambda}, \sigma_{B} e^{-i H_{0} \tau /} \sigma(t-\tau) e^{i H_{0} \tau / h}\right]\right]\right) .
\end{aligned}
$$

The trace operator $\operatorname{Tr}_{B}$ in eq. (2) indicates a summation over all the bath coordinates and $\sigma_{B}=\exp \left(-\mathrm{H}_{\mathrm{B}} /{ }^{\prime}{ }_{B} \mathrm{~T}\right) / \operatorname{Tr}_{B}\left[\exp \left(-\mathrm{H}_{B} / \mathrm{k}_{B} \mathrm{~T}\right)\right]$.

By substituting $\mathrm{H}_{\mathrm{I}}$ as defined explicitly in eq. (1) and then sum ning up all the bath coordinates, one can derive the following non-Markovian master equation for the reduced density matrix $\sigma(t)$

$$
\begin{aligned}
& \frac{d \sigma(t)}{d t}=-\frac{i}{h}\left[H_{S}, \sigma(t)\right] \\
& -\frac{1}{2} \int_{0}^{t} d \tau \cos \Omega \tau \operatorname{Tr}_{B}\left([F(0), F(-\tau)] \sigma_{B}\right)\left[X^{2}, e^{-i H_{g} \tau / /} \sigma(t-\tau) e^{i H_{g} \tau / h_{3}}\right] \\
& \left.+\frac{1}{2 M \Omega} \int_{0}^{t} d \tau \sin \Omega \tau \operatorname{Tr}_{B}\left([F(0), F(-\tau)] \sigma_{B}\right)\left[X, \mid P, e^{-i H_{s} \tau / h} \sigma(t-\tau) e^{i H_{g} \tau / h}\right)\right] \\
& -\frac{1}{2} \int_{0}^{t} d \tau \cos \Omega \tau \operatorname{Tr}_{B}\left(\{F(0), F(-\tau)] \sigma_{B}\right)\left[X,\left[X, e^{-i H_{s} \tau / /} \sigma(t-\tau) e^{i H_{s} \tau / /}\right]\right] \\
& +\frac{1}{2 M \Omega} \int_{0}^{t} d \tau \sin \Omega \tau \operatorname{Tr}_{B}\left(\{F(0), F(-\tau)] \sigma_{B}\right)\left[X,\left[P, e^{-i H_{s} \tau / h} \sigma(t-\tau) e^{i H_{s} \tau / h}\right]\right],
\end{aligned}
$$


where $F(-\tau)=\exp \left(-\mathrm{iH}_{B} \tau / \hbar\right) F \exp \left(\mathrm{iH}_{B} \tau / \hbar\right),[A, B]$ denotes a commutator and $\{A, B\}$ an anti-commutator. One can also show that for $F=\sum f_{k} q_{k}$,

$$
\begin{aligned}
& \operatorname{Tr}_{B}\left([F(0), F(-\tau)] \sigma_{B}\right)=-i \sum_{k=1}^{N}\left|f_{k}\right|^{2} \frac{\hbar}{m_{k} \omega_{k}} \sin \omega_{k} \tau \\
& \operatorname{Tr}_{B}\left([F(0), F(-\tau)\} \sigma_{B}\right)=\sum_{k=1}^{N}\left|f_{k}\right|^{2} \frac{\hbar}{m_{k} \omega_{k}}\left(2 n_{k}+1\right) \cos \omega_{k} \tau,
\end{aligned}
$$

and for $F=\Sigma f_{k} p_{k}$,

$$
\begin{aligned}
& \operatorname{Tr}_{B}\left([F(0), F(-\tau)] \sigma_{B}\right)=-i \sum_{k=1}^{N}\left|f_{k}\right|^{2} \hbar m_{k} \omega_{k} \sin \omega_{k} \tau \\
& \operatorname{Tr}_{B}\left(\{F(0), F(-\tau)\} \sigma_{B}\right)=\sum_{k=1}^{N}\left|f_{k}\right|^{2} \hbar m_{k} \omega_{k}\left(2 n_{k}+1\right) \cos \omega_{k} \tau,
\end{aligned}
$$

where $n_{k}=\left(\exp \left(\hbar \omega_{k} / k_{B} T\right)-1\right)^{-1}$.

\section{Discussion and conclusion}

With the master equation in eq. (5) one can calculate the equations of motion for the expectation value of position $\langle\mathrm{X}$; $\mathrm{t}\rangle$ and momentum $<P ; t>$. They are given by 


$$
\begin{aligned}
& \frac{d<X ; t\rangle}{d t}=\frac{\langle P ; t\rangle}{M} \\
& \frac{d\langle P ; t>}{d t}=-M \Omega^{2}\left\langle X ; t>+i \hbar \int_{0}^{t} d \tau \operatorname{Tr}_{B}\left([F(0), F(-\tau)] \sigma_{B}\right)\langle X ; t-\tau\rangle,\right.
\end{aligned}
$$

which indicates a change of the effective frequency from $\Omega$.

The Markovian master equation proposed by Caldeira and Leggett [8] can be expressed by

$$
\begin{aligned}
\frac{d \sigma}{d t}= & -\frac{i}{\hbar}\left[H_{S}, \sigma\right]-\frac{D}{\hbar^{2}}[X,[X, \sigma]] \\
& -i \frac{\gamma}{\hbar}\left([X, \sigma P]-[P, \sigma X]+\frac{1}{2}[(P, X\}, \sigma]\right. \\
= & -\frac{i}{\hbar}\left[H_{S}, \sigma\right]-\frac{D}{\hbar^{2}}[X,[X, \sigma]]-i \frac{\gamma}{\hbar}[X,\{P, \sigma \mid] .
\end{aligned}
$$

where the diffusion constant (in momentum space) $\mathrm{D}=2 \mathrm{M} \gamma \mathrm{k}_{\mathrm{B}} \mathrm{T}$. The equation of motion for $\langle\mathrm{X} ; \mathrm{t}\rangle$ and $\langle\mathrm{P}$; $\mathrm{t}\rangle$ is given by 


$$
\begin{aligned}
& \frac{d<X ; t>}{d t}=\frac{\langle P ; t>}{M} \\
& \frac{d<P ; t>}{d t}=-M \Omega^{2}\langle X ; t>-2 \gamma\langle P ; t>.
\end{aligned}
$$

Their results are not the Markovian limit of eqs. (5) and (8). One may mistakenly conclude that eq. (9) can be derived from eq. (5) using the following reasoning. The terms of $\sin \omega_{k} \tau$ and $\cos \omega_{k} \tau$ in $\operatorname{Tr}_{B}\left([F(0), F(-\tau)] \sigma_{B}\right)$ can be combined with $\sin \Omega \tau$ or $\cos \Omega \tau$ to form $\cos \left(\omega_{k} \pm \Omega\right) \tau$ or $\sin \left(\omega_{k} \pm \Omega\right) \tau$. For the case of $F=\sum f_{k} p_{k}$, if the counter-wave terms with $\omega_{k}+\Omega$ and those terms with $\sin \left(\omega_{k}-\Omega\right) \tau$ can be neglected, and then one can reduce eq. (5) to

$$
\begin{aligned}
& \frac{d \sigma(t)}{d t}=-\frac{i}{h}\left[H_{s}, \sigma(t)\right] \\
& -\frac{1}{h^{2}} \int_{0}^{t} d \tau D(\tau)[X,[X, \sigma(t-\tau)]]-\frac{i}{h} \int_{0}^{t} d \tau \gamma(\tau)[X,\{P, \sigma(t-\tau)\}],
\end{aligned}
$$

with

$$
\begin{aligned}
& D(\tau)=\hbar^{2} \sum_{k}\left|f_{k}\right|^{h \frac{h m_{k} \omega_{k}}{4}}\left(2 n_{k}+1\right) \cos \left(\omega_{k}-\Omega\right) \tau \\
& \gamma(\tau)=\frac{\hbar}{M \Omega} \sum_{k}\left|f_{k}\right|^{2} \frac{\hbar m_{k} \omega_{k}}{4} \cos \left(\omega_{k}-\Omega\right) \tau .
\end{aligned}
$$


Finally, if one makes the approximation of $\Sigma\left|f_{k}\right|^{2}\left(\hbar^{2} m_{k} \omega_{k} / 4 M \Omega\right) \cos \left(\omega_{k}-\Omega\right) t$ by a delta function as $\gamma \delta(t)$, and $\Sigma\left|f_{k}\right|^{2}\left(\hbar^{3} m_{k} \omega_{k} / 4\right)\left(2 n_{k}+1\right) \cos \left(\omega_{k}-\Omega\right) t$ by $D \delta(t)$, the above equation can then be reduced to eq. (9), a Markovian result of Caldeira and Leggett. However, because of the apparent non-equivalence between eq. (10) and eq. (8) in the Markovian limit, there must be a faulty argument in deriving eq. (11) from eq. (5). The problems can be traced back to the omission of the terms with $\omega_{k}+\Omega$ and at the same time making the approximation of $\exp \left(-\mathrm{iH}_{\mathrm{S}} \tau / \hbar\right) \sigma(\mathrm{t}-\tau) \exp \left(\mathrm{iH}_{\mathrm{S}} \tau / \hbar\right)$ by $\sigma(\mathrm{t}-\tau)$. Actually, the product of $\exp \left(-\mathrm{iH}_{\mathrm{S}} \tau / \hbar\right) \sigma(\mathrm{t}-\tau) \exp \left(\mathrm{iH}_{\mathrm{S}} \tau / \hbar\right)$ and $\sin \left(\omega_{\mathrm{k}}+\Omega\right) \tau$ or $\cos \left(\omega_{\mathrm{k}}+\Omega\right) \tau$ brings to $\sin \omega_{k} \tau$ or $\cos \omega_{k} \tau$ which can not be neglected.

Haake [7] has derived a non-Markovian master equation for the density matrix in the interaction representation based on a different type of interaction with $\mathrm{H}_{\mathrm{I}}=\Sigma\left(\mathrm{f}_{\mathrm{k}} \mathrm{ab}_{\mathrm{k}}{ }^{+}+\mathrm{f}_{\mathrm{k}}{ }^{*} \mathrm{a}^{+} \mathrm{b}_{\mathrm{k}}\right)$. Using eq. (3) and converting the creation and the annihilation operators into the position and the momentum operators, one can reformulate his equation in the following expression 


$$
\begin{aligned}
& \frac{d \sigma(t)}{d t}=-\frac{i}{\hbar}\left[H_{S}, \sigma(t)\right] \\
& +i \int_{0}^{t} d \tau \frac{M \Omega}{2 \hbar} \sum_{k}\left|f_{k}\right|^{2} \sin \left(\omega_{k}-\Omega\right) \tau\left[X^{2}+\frac{P^{2}}{M^{2} \Omega^{2}}, e^{-i H_{0} \tau / h} \sigma(t-\tau) e^{i H_{0} \tau / h}\right] \\
& -\int_{0}^{t} d \tau \frac{M \Omega}{2 \hbar} \sum_{k}\left|f_{k}\right|^{2}\left(2 n_{k}+1\right) \cos \left(\omega_{k}-\Omega\right) \tau\left(\left[X,\left[X, e^{-i H_{0} \tau / h} \sigma(t-\tau) e^{i H_{0} \tau / h}\right]\right]+\right. \\
& \left.\frac{1}{M^{2} \Omega^{2}}\left[P,\left[P, e^{-i H_{0} \tau / h} \sigma(t-\tau) e^{i H_{0} \tau / M}\right]\right]\right) \\
& -\frac{i}{\hbar} \int_{0}^{t} d \tau \sum_{k}\left|f_{k}\right|^{2} \cos \left(\omega_{k}-\Omega\right) \tau\left(\left[X, e^{-i H_{0} \tau / h} \sigma(t-\tau) e^{i H_{0} \tau / h} P\right]-\left[P, e^{-i H_{0} \tau / h} \sigma(t-\tau) e^{i H_{0} \tau / h} X\right]\right)
\end{aligned}
$$

The equation of motion for $\langle\mathrm{X}$; $\mathrm{t}\rangle$ and $\langle\mathrm{P}$; $\mathrm{t}\rangle$ is given by

$$
\begin{aligned}
& \frac{d<X ; t>}{d t}=\frac{\langle P ; t>}{M}-\int_{0}^{t} d \tau \sum_{k}\left|f_{k}\right|^{2}\left(\cos \omega_{k} \tau<X ; t-\tau\right\rangle+\frac{1}{M \Omega} \sin \omega_{k} \tau\langle P ; t-\tau>) \\
& \frac{d<P ; t>}{d t}=-M \Omega^{2}\left\langle X ; t>+\int_{0}^{t} d \tau \sum_{k}\left|f_{k}\right|^{2}\left(M \Omega \sin \omega_{k} \tau<X ; t-\tau>-\cos \omega_{k} \tau<P ; t-\tau\right\rangle\right)
\end{aligned}
$$

However, according to Kubo's phenomenological generalized Langevin theory [10] one has 


$$
\begin{aligned}
& \frac{d<X ; t\rangle}{d t}=\frac{\langle P ; t\rangle}{M} \\
& \frac{d<P ; t\rangle}{d t}=-M \Omega^{2}\left\langle X ; t>-2 \int_{0}^{t} d \tau \gamma(\tau)\langle P ; t-\tau\rangle .\right.
\end{aligned}
$$

The above equation describes a damped oscillator with a retarded frictional force proportional to the momentum whereas eq. (8) only shows a retarded frequency shift and eq. (14) shows both effects.

In conclusion, we have re-examined quantum Brownian motion of a harmonic oscillator. Using a different approach, a non-Markovian master equation for the density matrix is derived based on the interaction model previously employed by Caldeira and Leggett. Although using the same interaction model, our equation in the Markovian limit disagrees with the results of Caldeira and Leggett. The interaction model of Caldeira and Leggett shown here only leads to a retarded frequency shift, and Haake's model [7], however, leads only to underdamped oscillation. We have also shown that with such a model we could not derive Kubo's phenomenological generalized Langevin equation in eq. (15) involving a retarded friction proportional to the momentum.

The Born approximation used here assumes that the heat bath is sufficiently 
large and is never disturbed appreciably from its thermal equilibrium, i.e., it has a much shorter internal relaxation time than that of the oscillator, so any disturbance relaxes almost instantly. This assumption is valid if the heat bath is weakly coupled to the oscillator and the spectral distribution of the correlation function $\operatorname{Tr}_{B}\left(F(0) F(-\tau) \sigma_{B}\right)$ does not degenerate to extremely sharp and high lines [7].

The generalized Langevin equation of Kubo is a classical theory covering three possible regimes of motion: overdamped, critically damped and underdamped oscillation. If Kubo's theory is believed to be better than the master-equation approach used here, then a more realistic model other than those proposed earlier [7,8] is required; otherwise, one has to question if Kubo's generalized Langevin equation is an accurate description. As pointed out by Fox [11], the Gaussian assumption of Kubo's theory leads to a complete stochastic description in terms of a single, two-point correlation function in time. There one assumes that all high-order terms can be reduced to the second-order one. This assumption of the Gaussian property of the fluctuating driving force is questionable for a medium in which the Markovian assumption is no longer satisfied [12]. Also, the Kubo's generalized Langevin equation has neglected the 
quantum effects of the bath. Extension of this study to the overdamped regime of the non-Markovian quantum Brownian motion beyond the Born approximation by including all higher-order correlation functions deserves future investigation.

\section{Acknowledgement}

This work was supported by the U. S. Department of Energy, Office of Basic Energy Sciences, Division of Chemical Sciences, under Contract W-31109-Eng-38. I thank Dr. James R. Norris for initiating my interest in the Langevin model for solvent motion and related electron-transfer theory. 


\section{References}

[1] H. Dekker, Phys. Rev. A 16 (1977) 2116.

[2] I. R. Senitzky, Phys. Rev. 115 (1959) 227; 119 (1960) 670.

[3] F. Bloch and R. K. Wangsness, Phys. Rev. 89 (1953) 728.

[4] R. Zwanzig, J. Chem. Phys. 33 (1960) 1338.

[5] H. Mori, Prog. Theor. Phys. 33 (1965) 423.

[6] S. Nakajima, Prog. Theor. Phys. 20 (1958) 948.

[7] F. Haake, Z. Physik 223 (1969) 353; ibid. 364.

[8] A. O. Caldeira and A. J. Leggett, Physica 121A (1983) 587.

[9] R. P. Feynman and F. L. Vernon, Annals of Physics 24 (1963) 118.

[10] R. Kubo, Lectures in Theoretical Physics, Vol. 1 (Interscience, New York, 1959); Statistical Mechanics of Equilibrium and Non-equilibrium (North - Holland, Amsterdam, 1965).

[11] R. F. Fox, J. Math. Phys. 18 (1977) 2331; J. Stat. Phys. 16 (1977) 259.

[12] M. W. Evans, G. J. Evans, W. T. Coffey and P. Grigolini, Molecular Dynamics (John Wiley \& Sons, New York, 1982). 
